\title{
United States fatal pedestrian crash hot spot locations and characteristics
}

\author{
Robert J. Schneider \\ University of Wisconsin-Milwaukee \\ rjschnei@uwm.edu \\ Frank R. Proulx \\ Toole Design Group \\ fproulx@tooledesign.com
}

\author{
Rebecca L. Sanders \\ Arizona State University \\ rlsanders@asu.edu \\ Hamideh Moayyed \\ University of Wisconsin-Milwaukee \\ hmoayyed@uwm.edu
}

\begin{abstract}
US pedestrian fatalities are at their highest level in nearly three decades and account for an increasing share of total traffic fatalities $(16 \%)$. To achieve the vision of a future transportation system that produces zero deaths, pedestrian safety must be improved. In this study, we screened the entire US roadway network to identify fatal pedestrian crash "hot spot" corridors: 1,000-meter-long sections of roadway where six or more fatal pedestrian crashes occurred during an eightyear period. We identified 34 hot spot corridors during 2001-2008 and 31 during 2009-2016. While only five corridors were hot spots during both analysis periods, the 60 unique hot spots had remarkably consistent characteristics. Nearly all (97\%) were multilane roadways, with $70 \%$ requiring pedestrians to cross five or more lanes. More than three-quarters had speed limits of $30 \mathrm{mph}$ or higher, and $62 \%$ had traffic volumes exceeding 25,000 vehicles per day. All had adjacent commercial retail and service land uses, $72 \%$ had billboards, and three-quarters were bordered by low-income neighborhoods. Corridors with these characteristics clearly have the potential to produce high numbers of pedestrian fatalities. We also used hierarchical clustering to classify the hot spots based on their roadway and surrounding landuse characteristics into three types: regional highways, urban primary arterial roadways, and New York City thoroughfares. Each context may require different safety strategies. Our results support a systemic approach to improve pedestrian safety: Agencies should identify other roadway corridors with similar characteristics throughout the US and take actions to reduce the risk of future pedestrian fatalities.
\end{abstract}

Keywords: Pedestrian, fatalities, hot spots, multilane, high speed

\section{Article history:}

Received: June 17, 2020

Accepted: August 10, 2020

Available online: January 5,

2021

\section{Introduction}

Approximately 4,100 pedestrians died in US traffic crashes in 2009 (NHTSA, 2019a). While this loss of life should be unacceptable, it was still celebrated, as 2009 had the lowest-recorded number of pedestrian fatalities since the national Fatality Analysis Reporting System (FARS) was established in

Copyright Robert J. Schneider, Rebecca L. Sanders, Frank R. Proulx \& Hamideh Moayyed http://dx.doi.org/10.5198/jtlu.2021.1825

ISSN: 1938-7849 | Licensed under the Creative Commons Attribution - Noncommercial License 4.0

The Journal of Transport and Land Use is the official journal of the World Society for Transport and Land Use (WSTLUR) and is published and sponsored by the University of Minnesota Center for Transportation Studies. 
1975. However, since 2009, pedestrian fatalities have increased by more than $50 \%$ to approximately 6,300 in 2018. This is the highest level of annual fatalities in nearly three decades (NHTSA, 1993), and pedestrians now represent $16 \%$ of all fatal crash victims (NHTSA, 2019a). Reducing pedestrian fatalities is critical to achieving the vision of local, state, and national organizations to develop a transportation system that produces zero fatalities (Ecola, Popper, Silberglitt \& Fraade-Blanar, 2018; Toward Zero Deaths, 2011; Vision Zero Network, 2019). Safe streets for walking are also an essential ingredient for creating more livable and equitable communities.

Reducing pedestrian fatalities requires a thorough understanding of the problem. In this study, we screen the entire United States roadway network to identify fatal pedestrian crash "hot spots" (or "black spots")—specific roadway corridors where multiple fatal pedestrian crashes occurred during a relatively short period of time. Then we document common characteristics of these locations (motorist and pedestrian activity, adjacent land use, roadway design, and surrounding neighborhood demographic characteristics). Viewed systemically, hot spots can show planners, engineers, and other safety professionals the types of roadway corridors that are the most likely to experience high concentrations of pedestrian fatalities. By taking proactive steps, safety professionals can prevent similar locations from becoming future pedestrian fatality hot spots.

\section{$2 \quad$ Literature review}

The sections below describe common characteristics associated with pedestrian fatalities and spatial analysis approaches to identify high concentrations of traffic crashes.

\subsection{Factors associated with pedestrian fatalities}

Pedestrian fatalities are distributed unevenly throughout space and time and impact certain groups of people disproportionately. Broadly, pedestrian fatalities are more likely in areas where there are higher levels of pedestrian activity (Schneider, Vargo, \& Sanatizadeh, 2017). Overall, pedestrian injuries and fatalities tend to occur in urban areas (NHTSA, 2019b) and near land uses that generate more pedestrian activity, such as areas with commercial retail (Clifton \& Kreamer-Fults, 2007; Dumbaugh \& Li, 2010; Mansfield, Peck, Morgan, McCann, \& Tiecher, 2018; Wier, Weintraub, Humphreys, Seto, \& Bhatia, 2009). The character of these land uses may matter: big box stores and strip commercial centers increase pedestrian risk while pedestrian-scale retail decreases risk (Dumbaugh \& Li, 2010). The risk of being killed (fatalities per pedestrian crossing or per pedestrian trip) tends to be lower in locations with more pedestrian activity, a relationship known as "safety in numbers" (Geyer, Raford, Pham, \& Ragland, 2006; Jacobsen, 2003; Schneider, Vargo, \& Sanatizadeh, 2017). Pedestrian injuries and fatalities are also more prevalent on arterial roadways with higher traffic volumes, especially in urban areas (Mansfield et al., 2018; Stoker et al., 2015).

The physics of collisions make pedestrian fatalities more likely in certain circumstances. First, higher vehicle speeds are associated with higher pedestrian injury severity. Doubling the impact speed from $24 \mathrm{mph}(39 \mathrm{kmh})$ to $48 \mathrm{mph}(77 \mathrm{kmh}$ ) increases a pedestrian's risk of death from $10 \%$ to $75 \%$ (Tefft, 2013). The probability of death for elderly pedestrians is even higher across the range of speeds. The fact that arterial roadways are consistently associated with pedestrian fatalities (Dai, 2012; Mansfield et al., 2018; Rankavat \& Tiwari, 2013) may be because they facilitate higher-speed vehicle movement and because they are often wider and have more lanes, which increases pedestrian crossing time and complexity (Thomas et al., 2017). While the majority of minor-injury pedestrian crashes occur at roadway intersections, and four-way intersections may be riskier than three-way intersections (Dumbaugh \& Li. 
2010; Miranda-Moreno, Morency, \& El-Geneidy, 2011), most pedestrian fatalities occur at mid-block locations where vehicles are more likely to be traveling straight at higher speeds (NHTSA, 2019b). Sprawling development is also associated with pedestrian fatalities, since dispersed activity patterns often produce high levels of vehicle travel on extensive networks of high-speed roadways (Ewing, Schieber, \& Zegeer, 2003; Ewing \& Dumbaugh, 2009; Stoker et al., 2015). Second, larger vehicles are more likely to produce pedestrian fatalities (Jang et al., 2013; Lefler \& Gabler, 2003). The proportion of large vehicles (e.g., sport-utility vehicles) involved in pedestrian fatalities increased between 2013 and 2017 (GHSA, 2019).

Darkness increases the risk of pedestrian fatalities by reducing pedestrian visibility to drivers and decreasing available reaction time (Dai, 2012; Lee \& Abdel-Aty, 2005; Owens \& Sivak, 1993). It is also correlated with behaviors primarily associated with nighttime activities, such as alcohol usage and drowsiness (Lee \& Abdel-Aty, 2005; Owens et al., 2018; Sullivan \& Flannagan, 2001). Approximately $75 \%$ of US pedestrian fatalities occur in darkness (NHTSA, 2019b), and pedestrian crashes tend to produce more severe injuries in darkness and nighttime hours than during the daytime (Dai, 2012; Siddiqui, Chu \& Guttenplan, 2006), especially on weekend nights (Jang et al., 2013). Seasons with less daylight also tend to have more pedestrian fatalities (Griswold, Fishbain, Washington, \& Ragland, 2011; NHTSA, 2019b).

Roadway user behaviors are also associated with pedestrian fatalities. Nearly one-third of pedestrians killed had a blood-alcohol concentration of at least $0.08 \mathrm{~g} / \mathrm{dL}$ (NHTSA, 2019b) (note that this does not indicate fault; a person who was drinking may be killed while crossing legally in a crosswalk). Approximately $17 \%$ of drivers who killed a pedestrian had a blood-alcohol concentration above the legal limit (NHTSA, 2019b). This may underlie associations between pedestrian injuries and bar, club, and liquor store land uses (DiMaggio, Mooney, Frangos, \& Wall, 2016; Wedagama, Bird, \& Metcalfe 2006). As noted above, driver drowsiness is also likely a factor, albeit understudied, in collisions (Owens et al., 2018). Driver distraction (e.g., mobile phone use) was associated with an increasing number of pedestrian fatalities between 2005 and 2010 (Stimpson, Wilson, \& Muelleman, 2013). Injury severity is also positively correlated with pedestrians who are using a cell phone when hit (Jang et al., 2013).

Certain populations are more likely to be pedestrian fatality victims, including people living in poverty (Stoker et al., 2015). Approximately 70\% of pedestrians killed are male (NHTSA, 2019b). People over age 64 and people of color are also overrepresented (Jang et al., 2013; NHTSA, 2019b; Schneider, Vargo, \& Sanatizadeh ,2017; Smart Growth America, 2017). For example, relative to their share of the US population, people of color (particularly people who are Hispanic, African American, and Native American) represent a 54\% higher share of US pedestrian fatalities (Smart Growth America, 2017). Metropolitan regions with higher percentages of people born outside the US are also associated with higher pedestrian fatality rates (Schneider, Vargo, \& Sanatizadeh, 2017).

Pedestrian fatality risk can potentially be reduced through engineering (roadway design), education, and enforcement treatments. For example, pedestrian facilities such as sidewalks, median crossing islands, and pedestrian hybrid beacons have been shown to lower pedestrian crash risk (Blackburn, Zegeer, \& Brookshire, 2017; Stoker et al. 2015; Zegeer et al. 2013; Zegeer et al. 2016). Media coverage coupled with high-visibility police enforcement of crosswalk laws has increased driver yielding rates and reduced pedestrian crashes (Van Houten, Malenfant, Blomberg, \& Huitema, 2017). Automated speed enforcement has been found to significantly reduce speeds and may have potential to improve pedestrian safety (NHTSA, 2007). 


\subsection{Spatial analysis approaches}

Previous safety studies have identified locations with high concentrations of crashes, injuries, or fatalities. Most have used GIS to analyze the relative density of crash locations throughout communities, though their units of analysis and aggregation methods vary. Some approaches identify spatial clusters of crashes. These include kernel density (Jang et al., 2013; Morency \& Cloutier, 2006; Rankavat \& Tiwari, 2013; Schneider, Khattak, \& Zegeer 2002; Schuurman, Cinnamon, Crooks, \& Hameed, 2009), zone-based (e.g., census tract crash density) (Dumbaugh \& Li, 2010; Jang et al., 2013; Loukaitou-Sideris, Liggett, \& Sung, 2007; Mansfield et al., 2018), nearest neighbor clustering (Schneider, Ryznar, \& Khattak, 2004), and k-means clustering (Kim \& Yamashita, 2007). Other spatial approaches are tailored to identifying concentrations along roadway corridors, such as dynamic segmentation and moving window (Ambros, Havránek, Valentová, Krivánková, \& Striegler, 2016; Tang, Eftelioglu, Oliver, \& Shekhar, 2017).

Hot spot identification has limitations as an approach to prioritize high-risk locations in a roadway system. Notably, hot spots may only contain a small fraction of all traffic injuries in a community (Morency \& Cloutier, 2006). Further, since fatalities are relatively rare, hot spot locations tend to shift due to random variation over time. Several studies have reviewed methods for proactively identifying locations for safety improvements, and they recommend using systemic approaches to identify common roadway design features and crash types associated with crashes (using techniques like safety performance functions) and then applying countermeasures at locations with high-risk characteristics throughout a network (Ambros, et al. 2016; Elvik, 2008; Montella, 2010). Yet, to apply these methods, relevant explanatory variables are required across an entire roadway network. Unfortunately, the variety of variables needed to predict pedestrian fatalities with minimal accuracy are rarely available throughout an expansive system, such as all roadways at the state or federal level.

While detailed activity level, land use, roadway design variables are not available for the entire US roadway network, one way to begin to understand systemic patterns is to identify hot spot corridors and then explore several key variables within those corridors. If found, common hot spot characteristics can be viewed systemically as indicators of the types of locations that may be at risk for future pedestrian fatalities. While this has been done at the local level (City of Oakland, California, 2017; Solano County, California, 2018), to our knowledge, no other studies have identified the corridors with the highest concentrations of pedestrian fatalities across the entire US roadway network.

\section{$3 \quad$ Method}

We analyzed FARS data to identify the highest concentrations of pedestrian fatalities along the US roadway network, and then collected detailed information about each pedestrian hot spot corridor. From a systemic perspective, we recognize that hot spots are likely to shift over time (Thomas et al., 2018), so we conducted the same analysis approach for two separate eight-year time periods, 2001-2008 and 2009-2016. Partitioning the analysis into two time periods allowed us to determine if certain hot spots continued to have fatality problems over many years. It also allowed us to identify common characteristics of the hot spot corridors over time, even if specific locations shifted. These two periods also generally correspond with a decreasing trend (2001-2008) and increasing trend (2009-2016) in US pedestrian fatalities. The sections below detail our approach. 


\subsection{Pedestrian fatality data}

The FARS database is a census of all people killed in crashes involving a motor vehicle on public roadways in the US. This includes people who die within 30 days of a crash. FARS records indicate the type of roadway users involved in each fatal crash, including pedestrians. Like NHTSA, we excluded the 100 to 200 pedestrians per year who used personal conveyances (e.g., roller skates, electric scooters, wheelchairs, skateboards) (NHTSA, 2019b). See the FARS codebook for additional information (NHTSA, 2018).

FARS began including latitude and longitude coordinates of fatal crashes in 2001. Since the latest year with finalized data is 2016 , we analyzed geocoded fatal pedestrian crashes that occurred during the eight-year periods of 2001-2008 and 2009-2016.

Between 2001 and 2016, there were 76,951 pedestrian fatalities in the US. However, some of these fatalities occurred as a part of the same crash event. Since we wanted to identify geographic hot spots, we chose to use crash events rather than people killed as our unit of analysis. Doing so avoided assigning extra weight to the $1.4 \%$ of fatal crashes that involved multiple pedestrian fatalities, and resulted in an initial sample of 75,726 fatal pedestrian crashes. Next, we removed all 11,453 (15\%) of the fatal pedestrian crashes that occurred on freeways. From the remaining crashes, we removed 1,937 crashes that were not geocoded (3\%). This left 62,336 geocoded, non-freeway fatal pedestrian crashes. Of these, 29,939 occurred during 2001-2008, and 32,397 occurred during 2009-2016.

\subsection{Hot spot identification process}

We defined fatal pedestrian crash hot spot corridors as $1000 \mathrm{~m}$-long segments of roadway with six or more fatal pedestrian crashes during at least one eight-year analysis period. This is conceptually similar to a moving window approach. We chose six fatal pedestrian crashes within $1000 \mathrm{~m}$ as a threshold because it produced a reasonable number of hot spot corridors to analyze in the time available for this study. We considered any fatal crash within $50 \mathrm{~m}$ of the roadway centerline to be a part of the corridor. This distance captured the full width of the roadway right-of-way and a short distance on intersecting roadways influenced by the corridor. Identifying specific hot spot corridors was a multi-step GIS process, detailed in Appendix A.

\subsection{Hot spot data}

We gathered traffic, roadway design, roadway network, land use, and sociodemographic data for each corridor from publicly-available sources. Rather than measure each physical attribute precisely, which we recognized as inappropriate given our available resources and the historic nature of our analysis, our goal was to gather a variety of variables to provide a general sense of the character of each corridor. We observed roadway and land-use characteristics from free, online aerial and street-level imagery. Aerial images provided sufficient resolution to identify lanes, medians, crosswalks, parking lots, buildings, and building setbacks. Street-level images provided information about current posted speed limits, traffic signals and signs, and adjacent land uses. Our data are based on imagery from 2017 to 2019. While many corridors had similar characteristics throughout the 2001-2016 study period, we noted key changes by reviewing aerial images from the mid-2000s through 2019 (see notes in Appendix B). Approximately half of the corridors experienced changes in roadway lane striping, pedestrian crossing facilities, and/or sidewalks, though many of these changes were near the end of the study period or after 2016. We were unable to determine if the posted speed limit or other street-level characteristics changed in any of the corridors between 2001 and 2016. While these details should be explored in future studies, the avail- 
able data were sufficient to describe the general character of the hot spot corridors. To complement the imagery-based data collection, we also calculated various summary statistics of the road network within a one-half mile buffer of the hotspots using the OSMnx Python module to access OpenStreetMap data (Boeing, 2017).

\subsection{Cluster analysis}

Initial inspection of the hot spots suggested that there were distinct types of high pedestrian fatality corridors. Therefore, we used SPSS software to apply Ward's Method of hierarchical clustering to identify corridors with similar characteristics (IBM Corp., 2018). Specifically, we used z-scores to standardize values of 12 theoretically-important traffic and road design variables and 16 land-use variables (see variables in Appendix B) and used squared Euclidean distance to minimize the variation within clusters. We explored dividing the 60 corridors into two, three, and four clusters. Three clusters provided a dendrogram with distinct and intuitive corridor types (Figure 1).

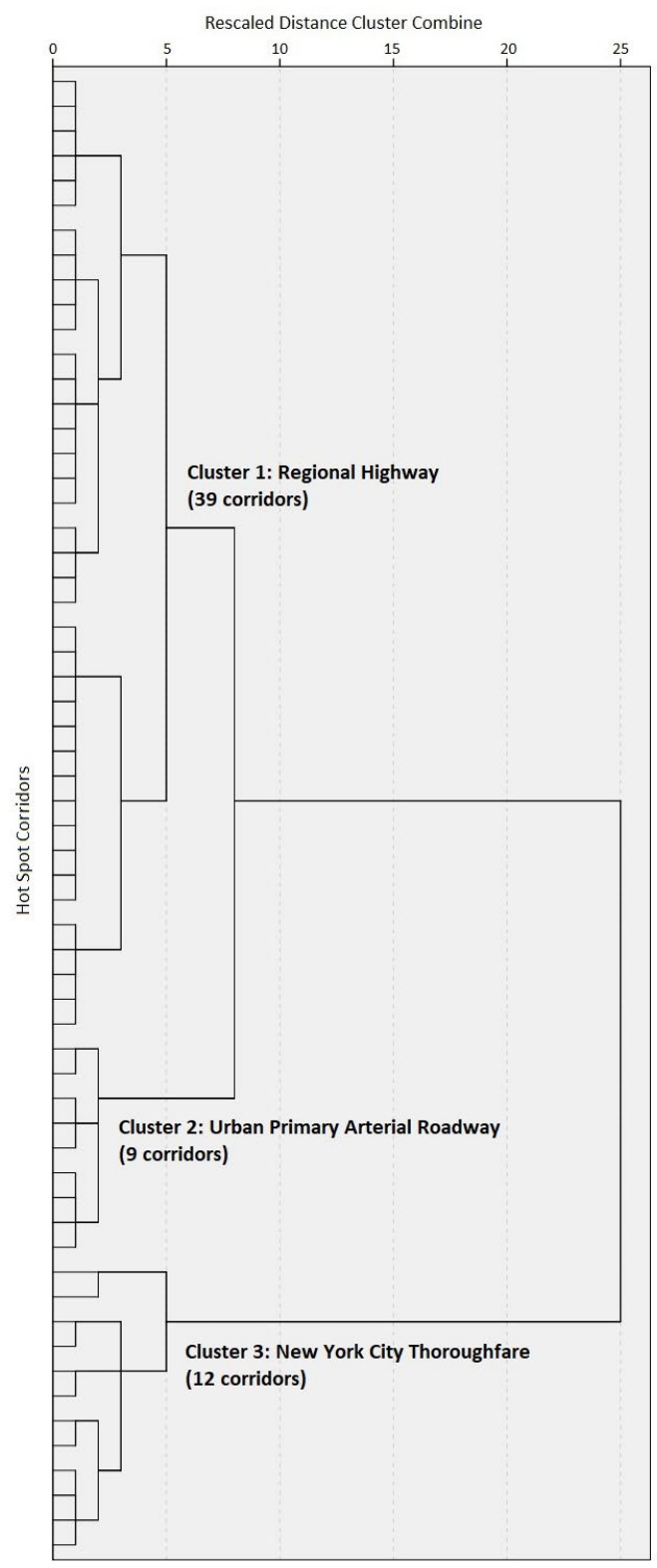

Figure 1. Hierarchical clustering dendrogram 


\section{$4 \quad$ Findings}

In total, we identified 60 unique $1000 \mathrm{~m}$ hot spot corridors with at least six fatal pedestrian crashes during either 2001-2008 or 2009-2016 (Appendix B). Thirty-four of these corridors were hot spots in 2001-2008 and 31 were hot spots in 2009-2016. Five were hot spots during both time periods. More than half of the unique hot spot corridors were in Sunbelt metropolitan regions, including Pasco County, Florida (7), Phoenix (4), Atlanta (4), Los Angeles (3), Miami (3), Orlando (3), West Palm Beach (2), Albuquerque, Bakersfield, Dallas, Jacksonville, Las Vegas, Pensacola, and Tucson. This result is consistent with other studies showing higher rates of pedestrian fatalities in the Sunbelt (Schneider, Vargo, \& Sanatizadeh, 2017). Many of these high-risk Sunbelt regions also have sprawling land-use patterns (Smart Growth America, 2014; Smart Growth America, 2017). Additionally, more than half of the unique hot spot corridors were part of the national or state highway systems, which may prioritize automobile speed and throughput.

Our cluster analysis found three distinct types of hot spot corridors (Table 1):

- Regional Highway (39 corridors). These tended to be high-speed, multilane roadways with high traffic volumes, many driveways, long block lengths, and relatively few pedestrian crossing opportunities (e.g., signalized intersections and additional pedestrian facilities). They often served adjacent properties with single-story commercial strip buildings and big-box stores set back behind large parking lots. Many were surrounded by relatively low-density residential areas and had low walk-to-work rates.

- Urban Primary Arterial Roadway (9 corridors). These hot spots had high speed limits and traffic volumes as well as multiple lanes, though these variable values tended to be smaller than for regional highways. They also had more signalized intersections, and some had additional pedestrian crossing facilities. Many had off-street parking associated with commercial retail and service uses, but the parking lots tended to be smaller than regional highways, and on-street parking was much more common. A distinctive characteristic of these hot spots was that many were in neighborhoods with incomes well below the regional average and majority Black and majority Hispanic populations.

- New York City Thoroughfare (12 corridors). These corridors had high traffic volumes, but only two were more than four lanes, and all had speed limits of $25 \mathrm{mph}(40 \mathrm{kmh})$. They had few driveway crossings and frequent signalized intersections. All of these corridors had on-street parking, and off-street parking was rare. Most were lined by multi-story buildings with first floor commercial retail and service uses and surrounded by neighborhoods with high population density and very high walk-to-work rates. All of these corridors were in New York City (though two corridors in New York City were in the urban primary arterial roadways cluster).

The sections below describe the 60 fatal pedestrian hot spot corridors in more detail. 


\begin{tabular}{|c|c|c|c|c|c|c|c|c|c|c|}
\hline \multirow{8}{*}{ 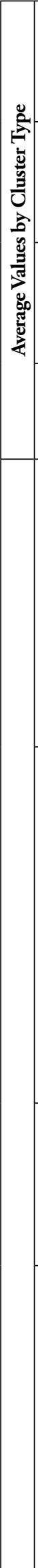 } & 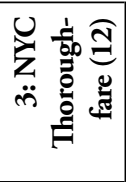 & $\vec{a}$ & 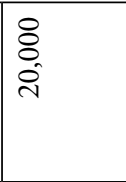 & $\stackrel{\Perp}{3}$ & $\stackrel{\infty}{\stackrel{0}{0}}$ & $\underset{+}{\stackrel{8}{\&}}$ & $\stackrel{\overbrace{}}{\tilde{c}}$ & $\underset{-}{\stackrel{-}{-}}$ & $\begin{array}{l}8 \\
\stackrel{\sim}{\sim}\end{array}$ & $\stackrel{\sim}{\varkappa}$ \\
\hline & 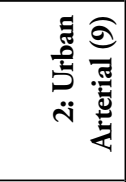 & $\infty$ & $\begin{array}{l}8 \\
\vdots \\
\stackrel{1}{1}\end{array}$ & $\stackrel{\Delta}{\circ}$ & तี & 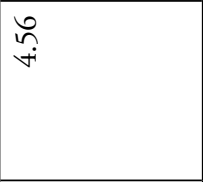 & $\vec{J}$ & $\begin{array}{l}\text { के } \\
0\end{array}$ & $\begin{array}{l}\hat{O} \\
\dot{m}\end{array}$ & $\begin{array}{l}\stackrel{\wp}{ } \\
\stackrel{\forall}{*}\end{array}$ \\
\hline & 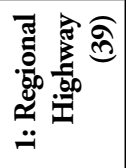 & $\stackrel{\infty}{\infty}$ & $\begin{array}{l}8 \\
\dot{\delta} \\
\dot{w}\end{array}$ & $\stackrel{\infty}{0}$ & $\stackrel{\hat{\imath}}{0}$ & $\overrightarrow{\tilde{\sigma}}$ & oे & $\stackrel{\infty}{0}$ & $\begin{array}{l}\stackrel{\infty}{\longrightarrow} \\
\stackrel{\forall}{F}\end{array}$ & $\underset{-}{\Omega}$ \\
\hline & 胥 & $\infty$ & $\begin{array}{l}\dot{8} \\
\dot{n} \\
\dot{n}\end{array}$ & $\hat{0}$ & $\hat{\tilde{o}}$ & $\tilde{\sim}$ & $\tilde{\kappa}$ & $\tilde{n}$ & $\begin{array}{l}\stackrel{\infty}{n} \\
\stackrel{n}{n}\end{array}$ & $\begin{array}{l}\infty \\
\infty \\
\dot{c}\end{array}$ \\
\hline & 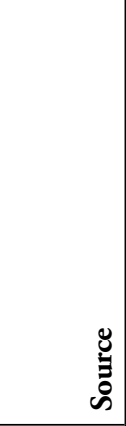 & 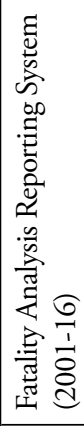 & 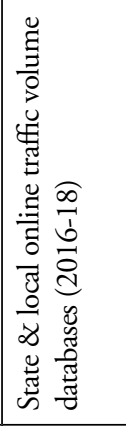 & 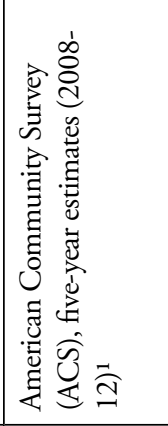 & 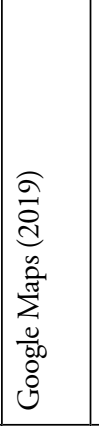 & 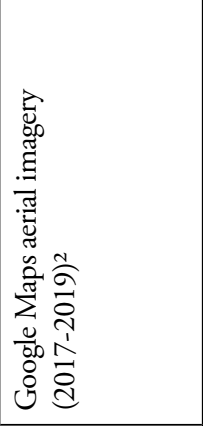 & 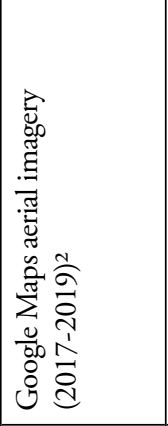 & 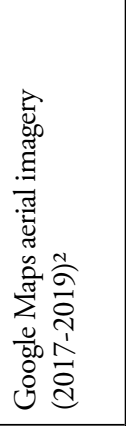 & 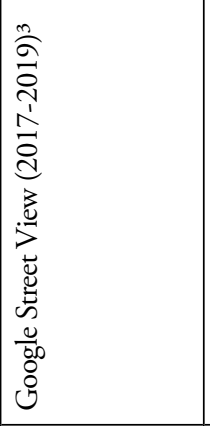 & 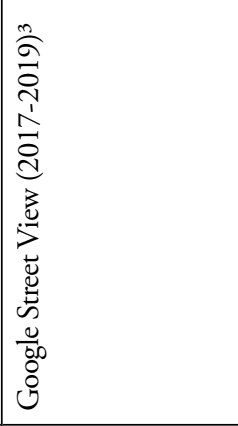 \\
\hline & 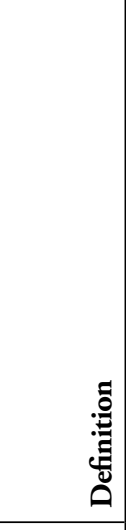 & 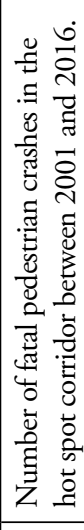 & 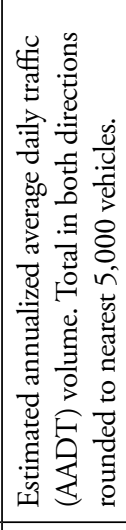 & 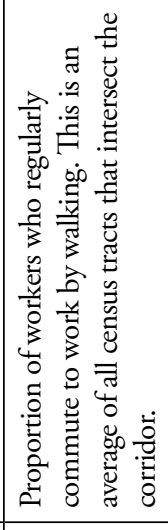 & 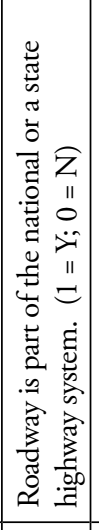 & 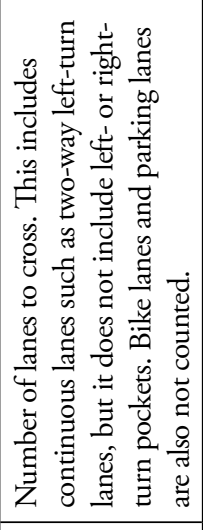 & 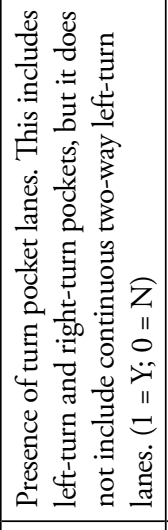 & 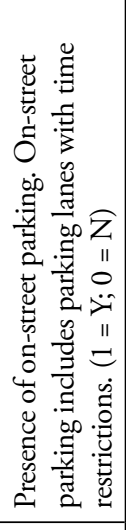 & 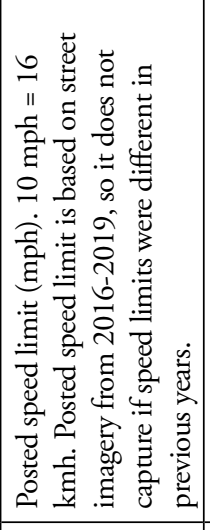 & 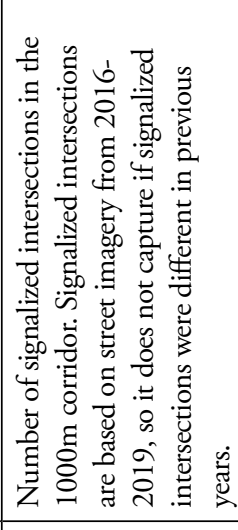 \\
\hline & 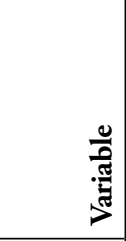 & 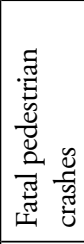 & 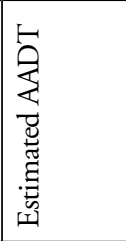 & 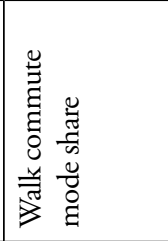 & 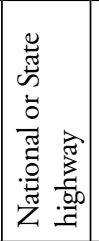 & 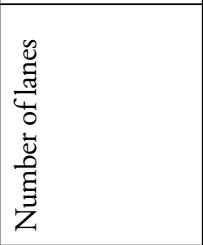 & 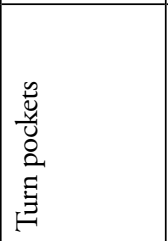 & 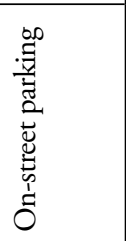 & 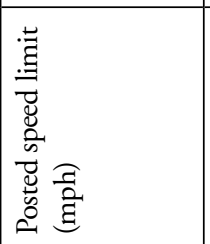 & 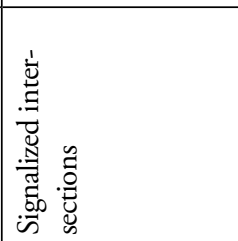 \\
\hline & & 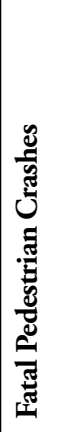 & 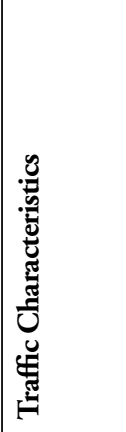 & & 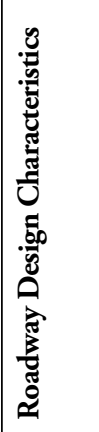 & & & & & \\
\hline
\end{tabular}




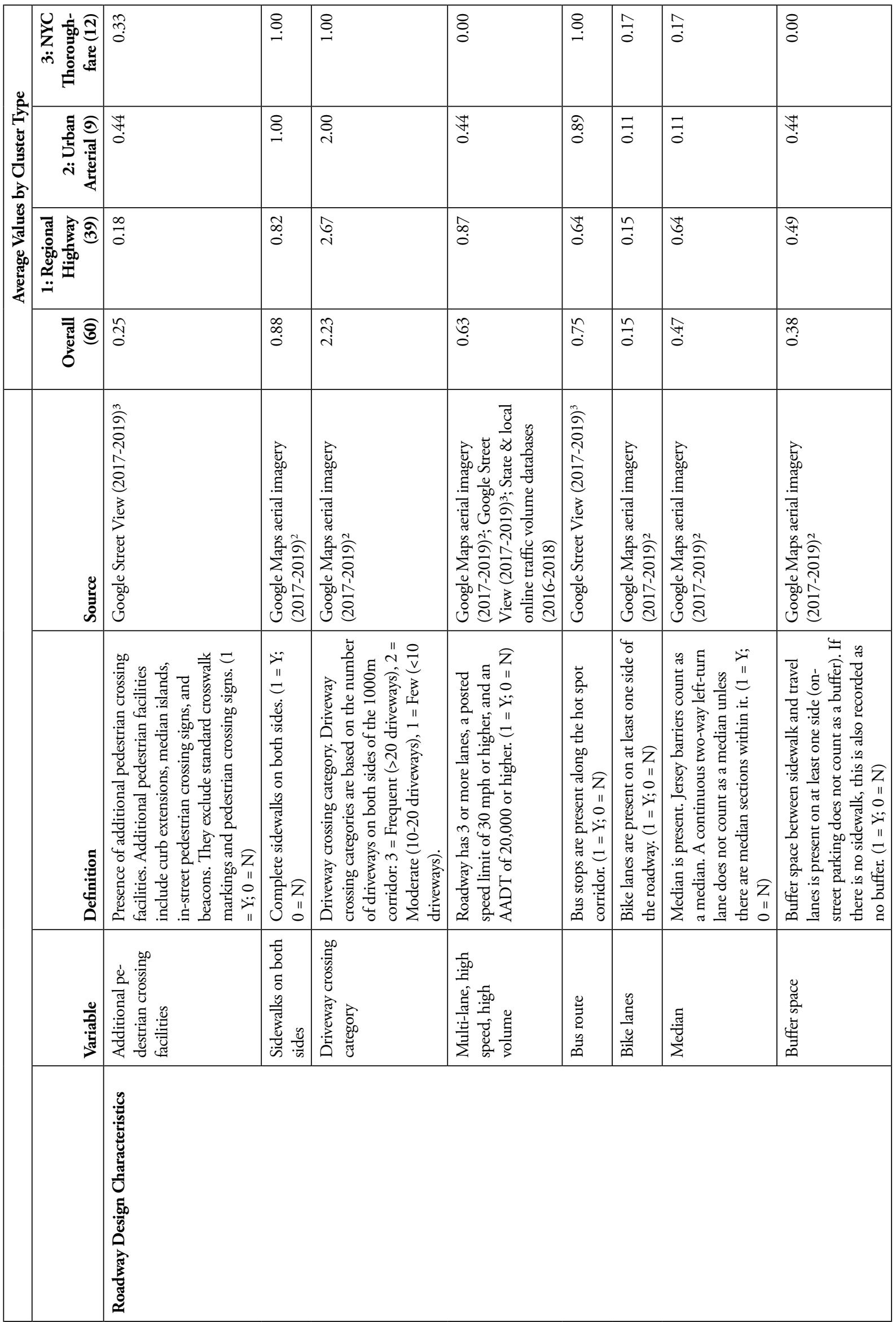




\begin{tabular}{|c|c|c|c|c|c|c|c|c|c|c|}
\hline 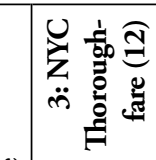 & के & $\overrightarrow{0}$ & $\stackrel{0}{\Xi}$ & 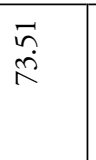 & $\begin{array}{l}\text { Iี } \\
\text { ḋ }\end{array}$ & 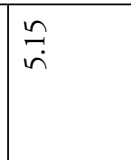 & 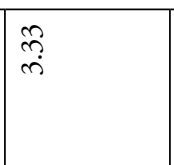 & $\begin{array}{l}\text { کे } \\
\text { ț }\end{array}$ & $\begin{array}{l}\overbrace{n}^{\infty} \\
\hat{n}\end{array}$ & $\begin{array}{l}\infty \\
\stackrel{\infty}{\infty} \\
\infty\end{array}$ \\
\hline 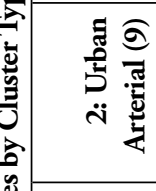 & \&̊+ & $\tilde{\tilde{n}}$ & $\begin{array}{l}\stackrel{f}{+} \\
\stackrel{+}{ \pm}\end{array}$ & $\begin{array}{l}0 \\
\text { Un } \\
\text { in }\end{array}$ & 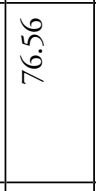 & $\vec{\sigma}$ & 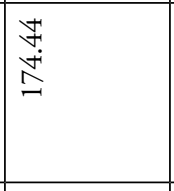 & $\begin{array}{l}\stackrel{8}{0} \\
\stackrel{4}{0}\end{array}$ & $\underset{\sigma}{\vec{b}}$ & 恋 \\
\hline 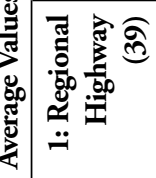 & 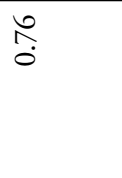 & đ̇ & 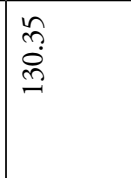 & 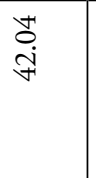 & $\begin{array}{l}\mathcal{N} \\
\stackrel{+}{*}\end{array}$ & $\begin{array}{l}\hat{\widehat{N}} \\
\text { ते }\end{array}$ & $\frac{\substack{n \\
\stackrel{\infty}{\infty}}}{\stackrel{\infty}{\infty}}$ & $\begin{array}{l}\hat{\infty} \\
\stackrel{\infty}{\leftrightarrow}\end{array}$ & \begin{tabular}{|l}
$\widetilde{O}$ \\
$\underset{\sim}{\mid}$
\end{tabular} & 常 \\
\hline 챙 & 菅 & గ్రి & $\stackrel{m}{\underset{I}{\sharp}}$ & ڤ̊. & 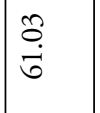 & $\begin{array}{l}\text { fे } \\
\stackrel{-}{-}\end{array}$ & $\mid \begin{array}{l}\hat{o} \\
\infty \\
i \\
i n\end{array}$ & 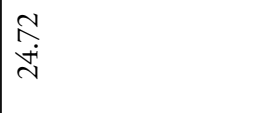 & $\begin{array}{l}\hat{\sigma} \\
\dot{\gamma}\end{array}$ & $\begin{array}{l}R \\
\stackrel{D}{9}\end{array}$ \\
\hline $\begin{array}{l}\mathscr{y} \\
\bar{\delta}\end{array}$ & 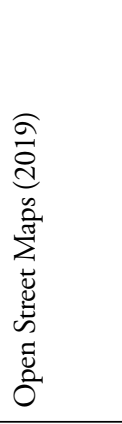 & 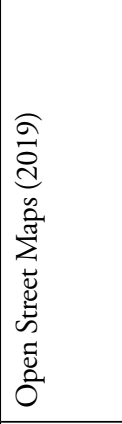 & 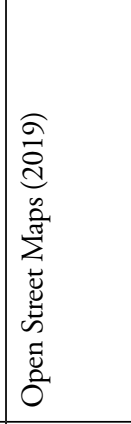 & 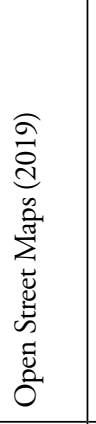 & 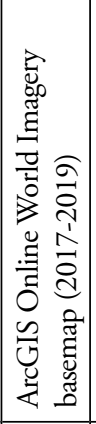 & 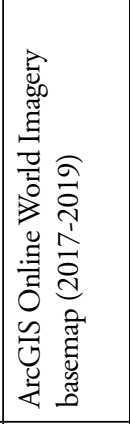 & 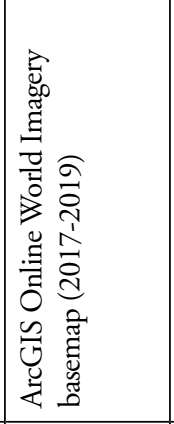 & 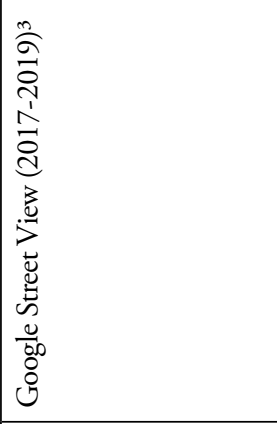 & 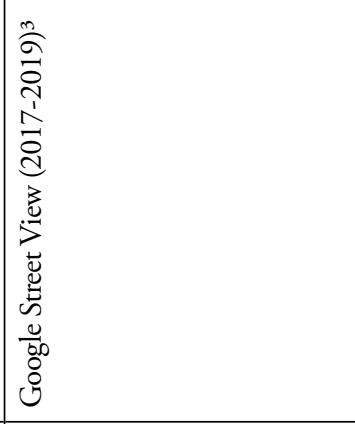 & 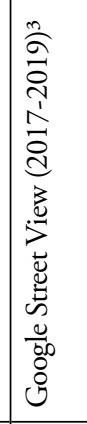 \\
\hline 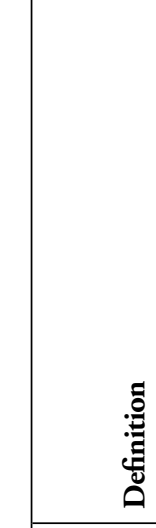 & 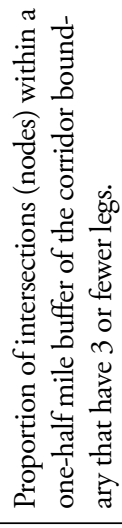 & 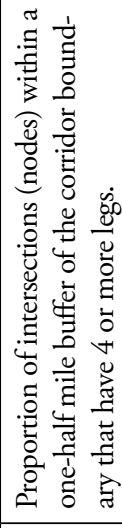 & 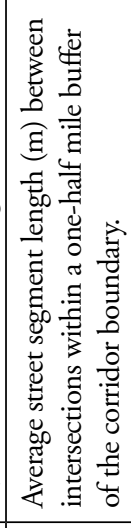 & 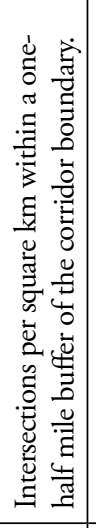 & 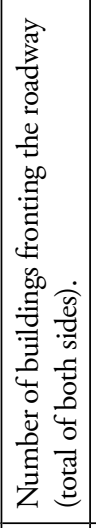 & 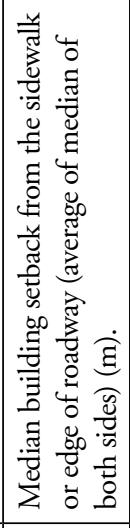 & 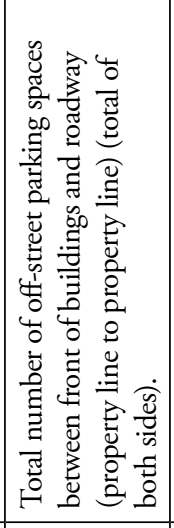 & 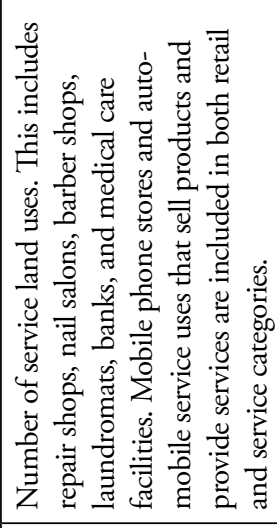 & 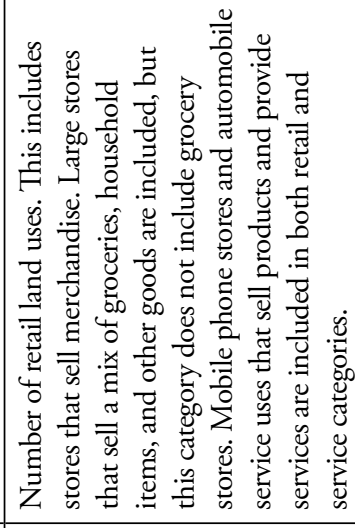 & 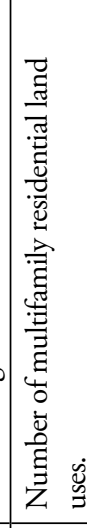 \\
\hline 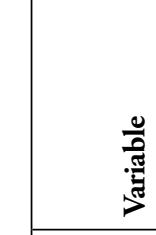 & 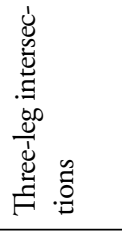 & 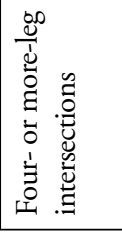 & 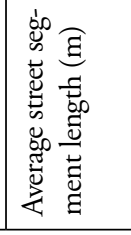 & 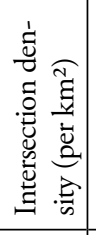 & 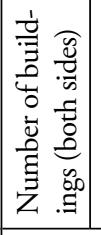 & 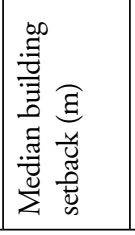 & 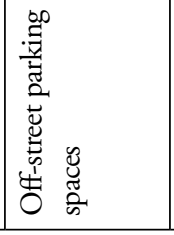 & 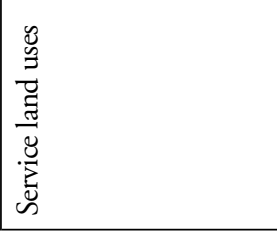 & 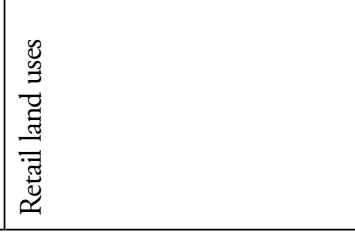 & 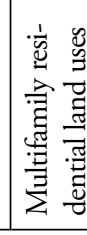 \\
\hline & \multicolumn{4}{|l|}{ 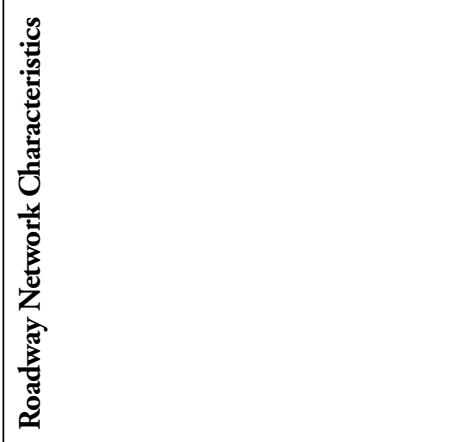 } & \multicolumn{6}{|l|}{ 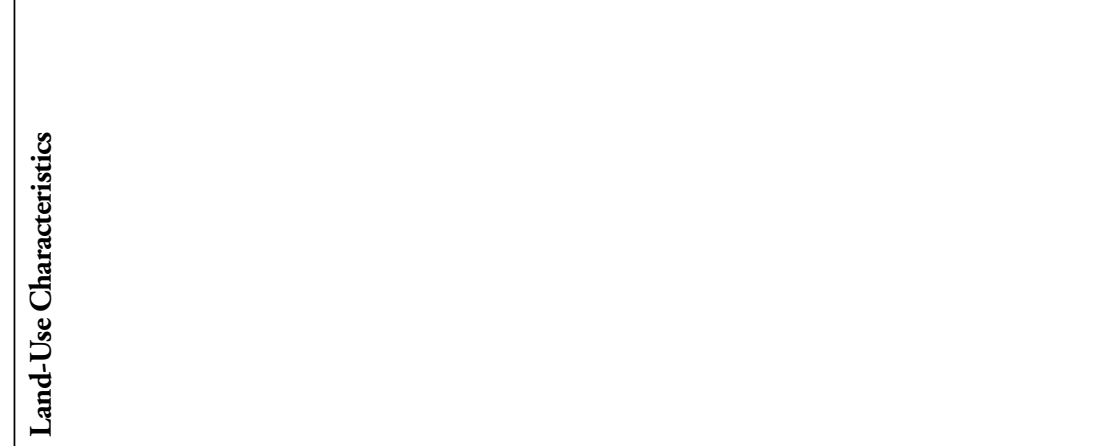 } \\
\hline
\end{tabular}




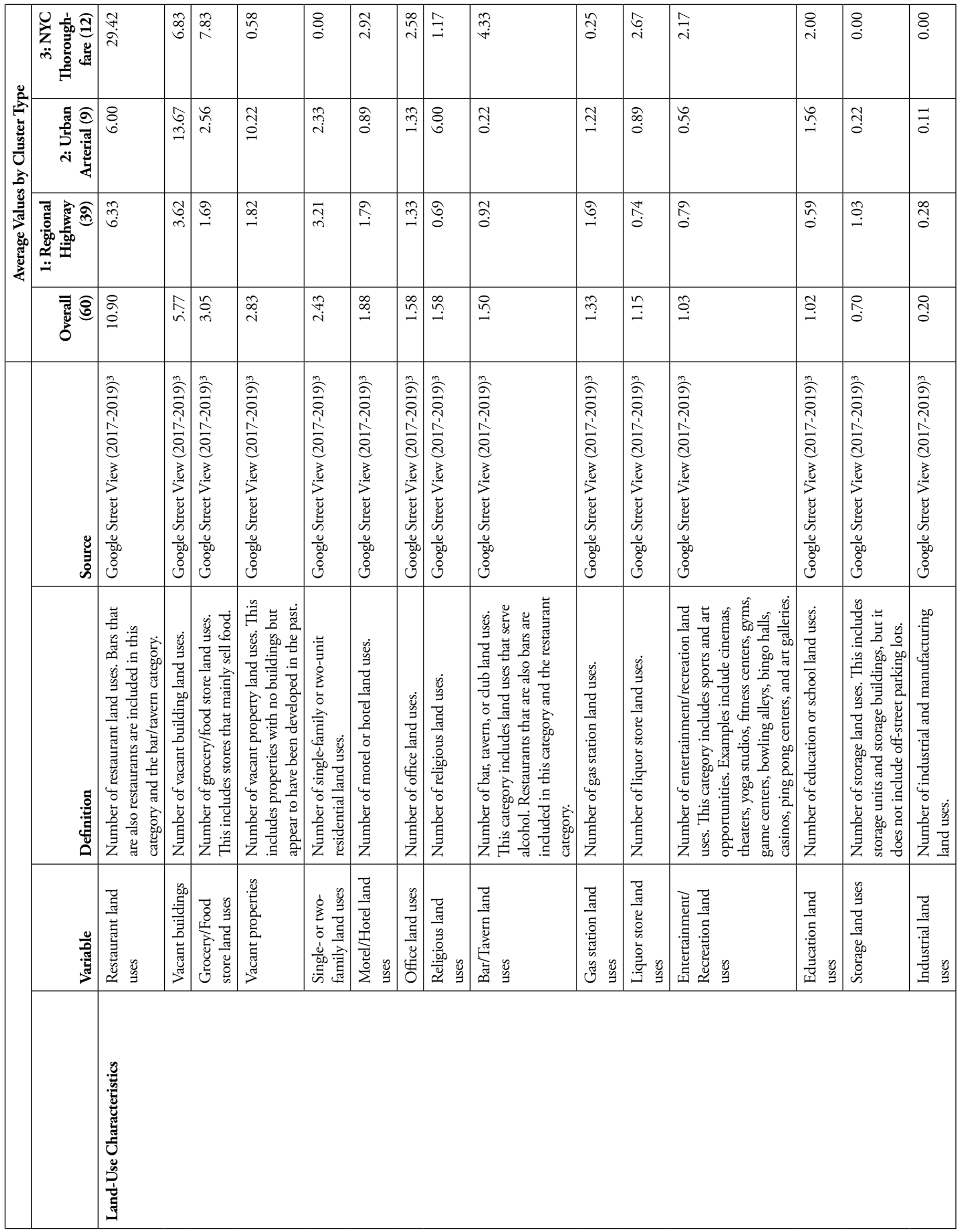




\begin{tabular}{|c|c|c|c|c|c|c|c|c|c|c|c|c|c|c|c|c|c|c|}
\hline & 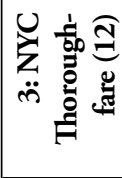 & 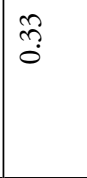 & $\begin{array}{c}\stackrel{\sim}{o} \\
\tilde{o}\end{array}$ & $\vec{~}$ & $\varkappa_{\delta}^{\infty}$ & $\stackrel{8}{8}$ & ఫ్రి & $\stackrel{8}{\rightarrow}$ & $\stackrel{0}{\circ}$ & : & 吕 & $\begin{array}{l}\tilde{\aleph} \\
\tilde{\aleph}\end{array}$ & 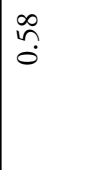 & io & $\stackrel{\infty}{\circ}$ & $\begin{array}{l}\text { ầ } \\
\text { on }\end{array}$ & $\stackrel{\curvearrowleft}{\rightleftharpoons}$ & $\stackrel{0}{\circ}$ \\
\hline 产 & 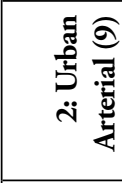 & 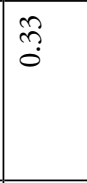 & $\overrightarrow{0}$ & ث્ & $\stackrel{0}{0}$ & 苦 & $\frac{8}{\gtrless}$ & สี & ֶิ & $\overrightarrow{0}$ & గ్రి & 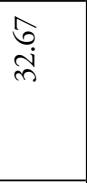 & $\stackrel{m}{0}$ & Oे & : & ત્રે & {$\left[\begin{array}{l}0 \\
0 \\
0\end{array}\right.$} & 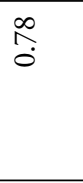 \\
\hline 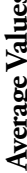 & 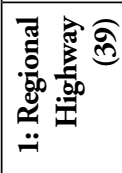 & $\stackrel{9}{0}$ & $\stackrel{\overbrace{}}{0}$ & $\stackrel{\overbrace{}}{\stackrel{\leftrightarrow}{+}}$ & {$\left[\begin{array}{c}\infty \\
\infty \\
\infty \\
\infty\end{array}\right.$} & 韋 & 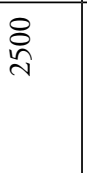 & $\stackrel{8}{0}$ & $\begin{array}{c}\infty \\
\infty \\
\infty \\
\infty\end{array}$ & స̂. & $\stackrel{m}{0}$ & $\begin{array}{l}\text { Oे } \\
\stackrel{\infty}{m}\end{array}$ & 菅 & $\stackrel{0}{\circ}$ & 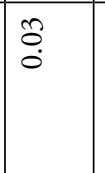 & 多 & 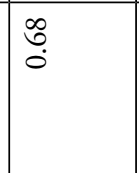 & 吕 \\
\hline & चี & $\stackrel{\infty}{0}$ & : & $\stackrel{n}{\vec{i}}$ & î. & $\hat{\hat{o}}$ & ఫू & శ్రి & $\stackrel{8}{\circ}$ & $\stackrel{\infty}{0}$ & ते & 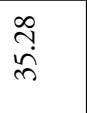 & 两 & त̃ & 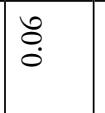 & के & $\mid \begin{array}{l}n \\
\hat{o}\end{array}$ & $\stackrel{n}{\circ}$ \\
\hline & : & 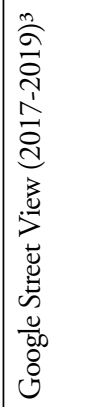 & 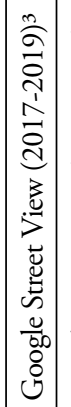 & 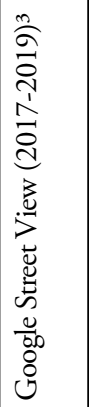 & 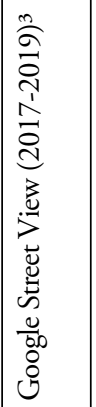 & 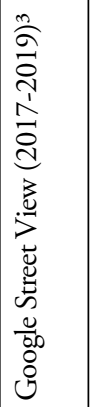 & 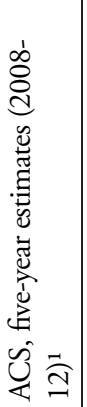 & & & 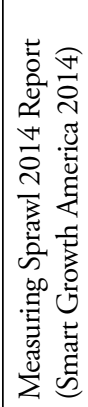 & 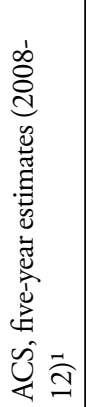 & 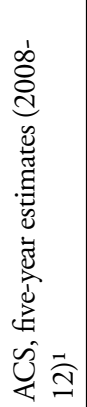 & 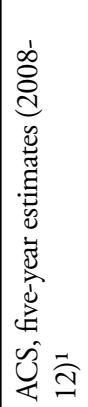 & 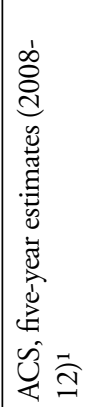 & 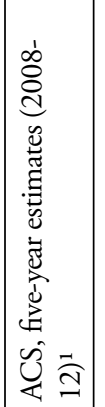 & 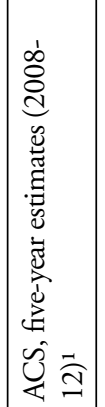 & 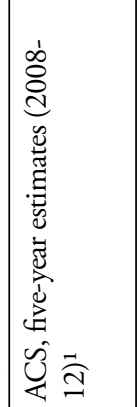 & 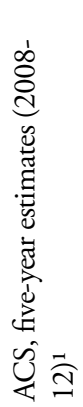 \\
\hline & $\begin{array}{l}\text { : } \\
\text { : }\end{array}$ & 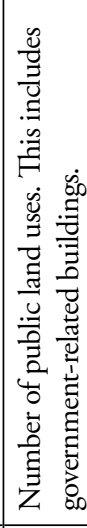 & 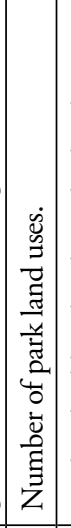 & 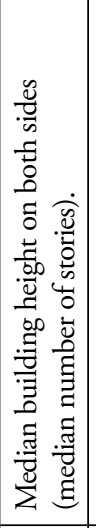 & 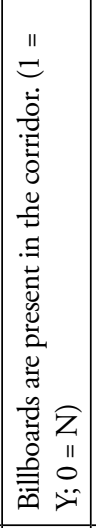 & 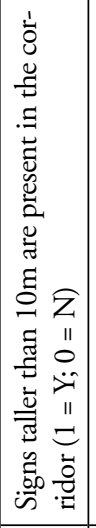 & 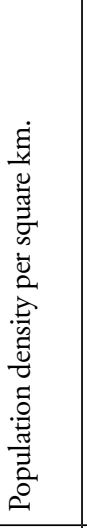 & 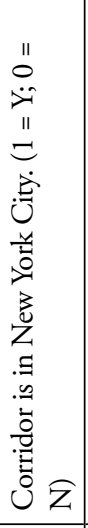 & 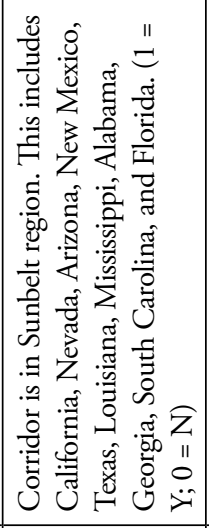 & 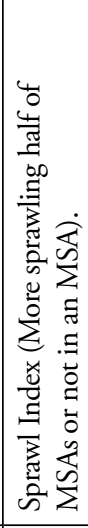 & 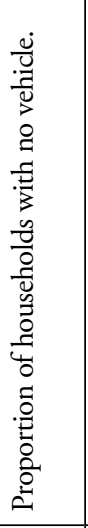 & 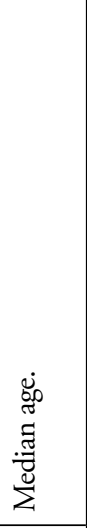 & 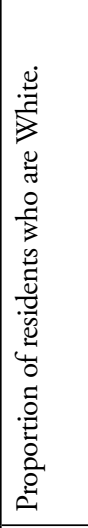 & 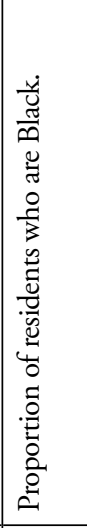 & 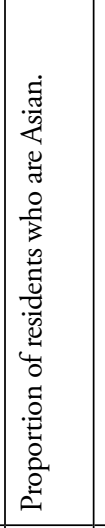 & 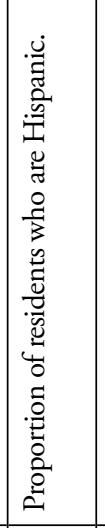 & 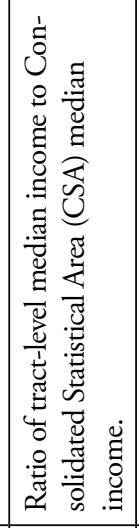 & 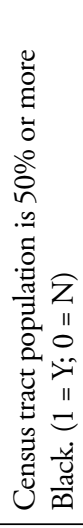 \\
\hline & 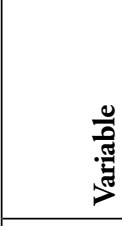 & 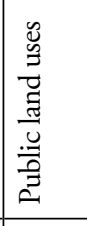 & 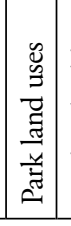 & 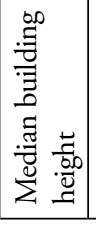 & 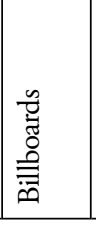 & 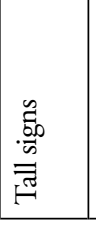 & 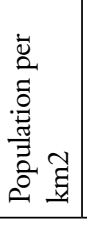 & 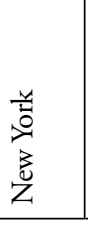 & 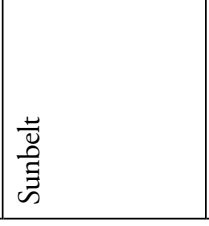 & 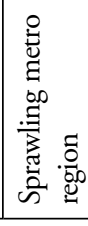 & 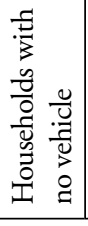 & 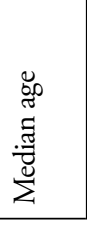 & 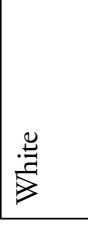 & 总 & $\frac{\frac{5}{9}}{4}$ & 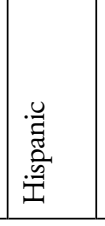 & 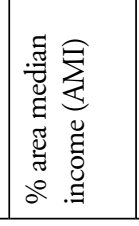 & 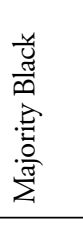 \\
\hline & & 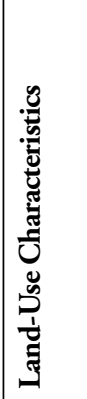 & & & & & & & & & 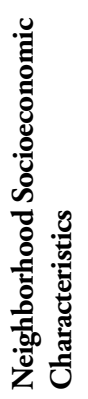 & & & & & & & \\
\hline
\end{tabular}



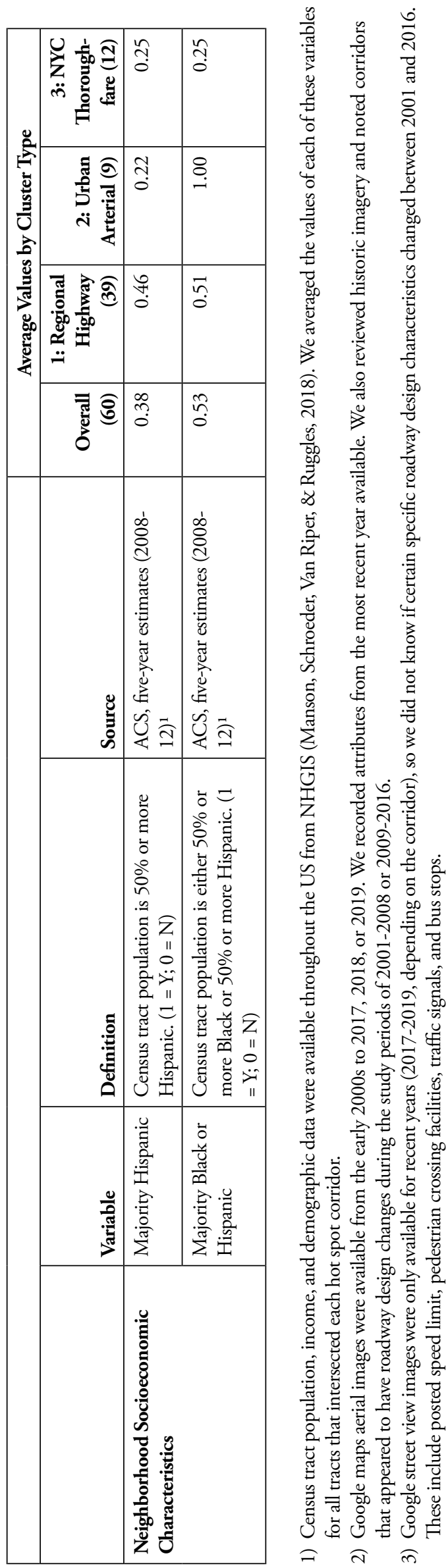


\subsection{Traffic and roadway characteristics}

Nearly two-thirds (63\%) of all hot spot corridors were roadways with 1) three or more lanes, 2) speed limits of $30 \mathrm{mph}(50 \mathrm{kmh}$ ) or higher and 3) high traffic volumes (more than 20,000 AADT) (Appendix B). Most regional highway corridors (87\%) had all three characteristics. Across all 60 corridors:

- $58(97 \%)$ had three or more lanes for pedestrians to cross (pocket turn lanes and parking lanes are not counted).

- $42(70 \%)$ had five or more lanes for pedestrians to cross (4-lane roadways with an additional two-way left-turn lane are included).

- $12(20 \%)$ had seven or more lanes for pedestrians to cross (6-lane roadways with an additional two-way left-turn lane are included).

- $46(77 \%)$ had speed limits of $30 \mathrm{mph}$ or higher. Only the 14 New York City corridors had speed limits of $25 \mathrm{mph}(40 \mathrm{kmh})$.

- $30(50 \%)$ had speed limits of $40 \mathrm{mph}(64 \mathrm{kmh})$ or higher.

- 37 (62\%) had traffic volumes over 25,000 AADT.

- 14 (23\%) had traffic volumes over 50,000 AADT.

Approximately half of the corridors had additional pocket lanes for left turns, right turns, or both, though turning pockets were the most common along regional highways. Nearly all of the urban arterial and New York City thoroughfares had on-street parking, while only one regional highway had this feature. Pedestrian crossing opportunities were relatively infrequent in certain corridors, including 34 (57\%) with two or fewer signalized intersections over 1000m (all but one of these corridors was a regional highway). One-quarter of the corridors included additional pedestrian crossing facilities (e.g., pedestrian hybrid beacons, rapid flashing beacons, in-street yield to pedestrian signs) at unsignalized locations, and some of these facilities were added after many pedestrian fatalities occurred (Appendix B). Three-quarters of the corridors were along bus routes, which are likely to generate pedestrian activity when bus service is operating. Stopped buses may make it difficult for drivers and pedestrians to see each other in some locations, and pedestrians may take additional risks when crossing to avoid missing a bus. Few (15\%) of the hot spots had bike lanes, further suggesting that the hot spot corridors primarily prioritize automobile movement.

Urban primary arterial and New York City thoroughfare hot spot corridors all had sidewalks on both sides. They also tended to have fewer driveway crossings and were more likely to be in neighborhoods with greater street connectivity (e.g., shorter block lengths, higher intersection density, and more four-way intersections) than rural highway corridors.

The hot spot corridors had a variety of pedestrian activity levels. The New York City thoroughfares were in census tracts with an average walk commute mode share of $24 \%$ compared with just $4 \%$ for urban primary arterial and 3\% for regional highway corridors. Street view imagery suggested particularly low pedestrian volumes along most regional highway corridors. This is somewhat surprising since pedestrian crashes tend to happen during a very small fraction of all pedestrian crossings, and pedestrian fatalities are even rarer; thus, hot spots are expected to have a relatively high number of pedestrian crossings. Strikingly, each regional highway corridor hot spot produced a similar number of pedestrian fatalities as the New York City thoroughfares that had eight times the walk commute mode share (which likely underestimates the difference in overall pedestrian activity between these types of corridors). This suggests an incredibly high risk to the few pedestrians who do walk along and cross regional highway corridor roadways. This preliminary finding warrants a more detailed analysis of pedestrian crash rates based on actual counts. 


\subsection{Land-use characteristics}

Land-use patterns in the hot-spot corridors, particularly the regional highway corridors, are automobileoriented. The regional highway corridors have an average median building setback of $22 \mathrm{~m}$ and more than 800 off-street parking spaces in lots fronting on the corridor. Most regional highway corridors have one-story buildings. Nearly three-quarters $(72 \%)$ of the 60 hot spot corridors have billboards, objects most often designed to capture driver attention. Billboards are most common along regional highway hot spots (85\%) but are still prominent along urban principal arterials (67\%) and New York City thoroughfares (33\%).

The most common land uses in all types of hot spot corridors are service ( 24.7 per corridor) and retail (24.0) (Table 1). These uses were often found in malls, strip shopping centers, and multi-unit building storefronts. Restaurants (10.9), vacant buildings (5.8) and grocery/food stores (3.1) were also common. Both vacant buildings and vacant properties were most prominent in urban primary arterial hot spots, which may suggest economic hardship in these corridors. Interestingly, bars/taverns were in half and liquor stores were in nearly two-thirds of the corridors, but few corridors had high concentrations of these alcohol-oriented uses (only two corridors in Manhattan had more than seven bars/taverns). While none of the corridors were exclusively residential, many corridors had high concentrations of multi-family residential buildings (13.7 per corridor).

\subsection{Neighborhood socioeconomic characteristics}

Socioeconomic data showed that many neighborhoods surrounding the 60 hot spot corridors had low incomes and had high proportions of people of color.

- 53 (88\%) had median household incomes lower than the area median income (AMI). 45 (75\%) had median household incomes lower than $75 \%$ of the AMI. The New York City thoroughfares were an exception since more than half had incomes higher than the AMI.

- $23(38 \%)$ had a majority of residents who were Hispanic (16\% of the 2010 US population was Hispanic). Nine (15\%) had a majority of residents who were Black (13\% of the US population). 32 (53\%) had a majority of residents who were either Hispanic or Black (39\% of the US population). Urban primary arterial hot spots were strongly associated with residents who were Black, and all were in neighborhoods that were either majority Black or majority Hispanic.

\subsection{Changes over time}

We expected that the hot spot locations identified during 2001-2008 and during 2009-2016 would be somewhat different due to the statistical phenomenon of regression to the mean (AASHTO, 2010). Additionally, other systemic changes could influence shifts in hot spot locations. For example, we found that the percentage of Sunbelt hot spot corridors increased from 56\% (19 of 34) in 2001-2008 to 68\% (21 of 31) in 2009-2016. In contrast, the number of hot spot corridors in New York City decreased from $32 \%$ (11 of 34 ) to $13 \%$ ( 4 of 31 ). We do not have sufficient data to determine the causes of these shifts, but continued population growth in the Sunbelt, changes in corridor traffic volumes, and systemic transportation system changes in New York City (e.g., multiple corridor redesign projects since 2008; citywide speed limit lowered to $25 \mathrm{mph}$ in 2014) are potential factors.

We are unable to determine from our data whether or not the pedestrian crossing facilities that were added in specific corridors during the study periods prevented additional pedestrian fatalities from occurring. However, well-developed pedestrian crash reduction factors for several of the treatments used at uncontrolled crossing locations suggest that this is likely (Zegeer et al., 2016). 


\subsection{Pedestrian safety problems along longer corridors}

Several hot spot corridors were at separate locations along the same thoroughfare, indicating serious pedestrian problems along a much longer section of roadway. These include 27th Street in Phoenix, Arizona (two corridors) and US Highway 41 in South Bradenton, Florida (two corridors).

However, US Highway 19 in Pasco County, Florida stood out with seven hot spot corridors. Expanding our search area, we found that this 20 -mile stretch of highway experienced 89 fatal pedestrian crashes (90 fatalities) during 2001-2008 and another 46 fatal pedestrian crashes (47 fatalities) during 2009-2016. For reference, the 137 pedestrian fatalities in this 20-mile corridor during 2001-2016 is nearly equal to the total number of fatalities on commercial airlines in the entire US between 2003-2018 (161) (NTSB, 2019).

\section{Discussion}

As a whole, the characteristics of the 60 unique fatal pedestrian crash hot spot corridors highlight two issues that planners, engineers, and other safety professionals must take seriously in order to create a future transportation system that produces zero deaths. These issues include physical design and social equity.

\subsection{Physical design: Shift from automobile mobility to multimodal accessibility}

The fatal pedestrian crash hot spot corridors have remarkably consistent design characteristics. The majority of US roadways have two travel lanes, but nearly all of the hot spot corridors were on multilane roadways (the only two-lane hot spot corridors were streets in New York City with high pedestrian activity levels). With the exception of New York City thoroughfares, most of the hot spot corridors require pedestrians to cross five or more lanes. Most of these corridors also have high automobile volumes and high posted speed limits, suggesting that they are designed primarily as thoroughfares for automobile traffic. While the specific hot spot corridors identified in 2001-2008 and 2009-2016 may not experience a high number of pedestrian fatalities in the next eight years, they have the ingredients to continue to produce these types of traffic deaths. Importantly, there are many other roadway corridors with similar characteristics throughout the US that may become the next hot spots. If we take the message of "zero deaths" seriously, these types of locations deserve significant attention and resources to prevent future pedestrian fatalities. At a minimum, our results should cause transportation agencies to conduct road safety audits and rethink their design practices and policies related to pedestrian crossing opportunities and traffic speed along similar corridors.

Considering surrounding community context, most of the fatal pedestrian crash hot spot corridors fall into two general categories: 1) thoroughfares that provide automobile mobility through major city neighborhoods (urban principal arterials and New York City thoroughfares) and 2) high-speed thoroughfares surrounded by development designed almost exclusively for automobile use (regional highway corridors). The potential to improve pedestrian safety in each of these two groups of corridors

is likely to be different, and both cases require individual communities and metropolitan regions to rethink their emphasis on using these corridors primarily for automobile mobility. 


\subsubsection{Thoroughfares within major city neighborhoods (Urban principal arterial and New York City thoroughfare hot spots)}

The first category of hot spots includes street corridors in the cities of New York, Los Angeles, Philadelphia, Miami, Detroit, and St. Louis. In many cases, they were originally neighborhood-oriented commercial streets, but they now serve as some of the main automobile routes through these cities.

Fortunately, these corridors have good potential for improvement due to their urban contexts. First, speed limits can be reduced and streets can be redesigned for slower traffic (e.g., remove travel lanes, narrow travel lanes, add curb extensions and median islands, reduce corner turning radii). Dense, mixed-use cities have high levels of accessibility, so people and goods do not need to travel as far or as fast between destinations. Second, some automobile trips should be shifted to other modes to reduce traffic volumes. Most of these historic cities have excellent public transit systems, are expanding low-stress bicycle networks, and are supporting micromobility options (e.g., shared e-bikes and e-scooters); in these cases, travel lanes can be repurposed as expanded sidewalks and/or bike or mixed-mobility lanes. As traffic volumes decrease and these thoroughfares are redesigned, they will become even safer for walking, further shifting the emphasis toward multimodal accessibility. The physical structures of these historic cities also provide a well-connected street grid, which can allow automobile traffic to diffuse across the network.

While these hot spot corridors have high potential for improvement, they are unfortunately less common than the second category of hot spots.

\subsubsection{High-speed thoroughfares and surrounded by development designed for automobile use (regional highway hot spots)}

The second category of hot spots includes corridors that are mainly in suburban parts of metropolitan regions. Many of these roadways were established to provide high-speed, high-capacity automobile movement and connect with outlying communities or other metropolitan regions. They were often expanded as large-scale, automobile-oriented land uses developed on adjacent properties. Few of these corridors were originally designed with the intention to make pedestrian travel convenient or safe.

While some of these corridors have been retrofitted in recent years with enhanced pedestrian crossing facilities, their basic physical layout with long distances between destinations, scant crossing opportunities, and wide roadways serving large volumes of high-speed automobile traffic makes walking unappealing. The potential for substantial improvement would require major changes to the character of the roadway and the surrounding development. Slowing vehicle speeds is critical for pedestrian safety, so narrowing the roadway and lowering speed limits should be considered and potentially coupled with automated speed enforcement. Traffic volumes should also be reduced by shifting automobile trips to other modes. Repurposing excess lanes as physically-separated, shared mobility lanes (e.g., for bikes and e-scooters) could help change the character of the corridor and encourage mode shift. However, to make these design changes feasible in areas that are currently so highly automobile-dependent, the distances between destinations in the surrounding communities need to be reduced. This could be done by redeveloping large parking lots into walkable mixed-use developments, establishing high-quality public transit services, and enhancing freight rail connections between regions. These changes are likely to take strong political leadership and decades to implement. Nonetheless, to create a transportation system with zero pedestrian fatalities, planners must outline a comprehensive and long-term vision that includes overhauling these types of corridors. In the short-term, strategies such as pedestrian hybrid beacons and additional signalized crossing locations should be used to the fullest extent possible to save lives now. 


\subsection{Social equity: Make roadway corridors safe for all}

The fatal pedestrian crash hot spot corridors tend to be in lower-income areas, especially among regional highway and urban principal arterial hot spots. In many cases, the hot spot corridor is the central place where local residents work, shop, socialize, and worship. Some of these people do not own cars, so they may not have an option to travel these corridors in the relative safety of an automobile. It is unacceptable for certain groups of people to have a greater chance of being killed because their daily activities are located along a corridor designed with automobile mobility prioritized over pedestrian safety.

Since more than half of the hot spot corridors pass through communities with either predominantly Black or predominantly Hispanic residents, it is important to make sure that these groups are represented in transportation planning processes. If not, it is essential to go to them directly and listen to what they need and want for their neighborhoods. In some cases, local drivers and pedestrians may not understand certain traffic signs due to language barriers, or there may be different cultural expectations for how roadways are used.

\section{Considerations and future research}

Conducting a nationwide analysis presents a variety of data challenges. For example, certain variables that may be associated with pedestrian fatalities were not available for this analysis. These include pedestrian volumes, actual travel speeds, roadway lighting, and behaviors (e.g., speeding, red-light-running, crossing against a traffic signal). We explored pedestrian volumes using street-level imagery, but this only represents pedestrian activity at a very specific time when the images were captured. We also reported census tract walk commute estimates, but these only represent one trip purpose (people who walk to work regularly). Given the limited extent of pedestrian counts available in the US, pedestrian exposure data continues to be a major research need, especially to quantify pedestrian risk in hot spot corridors.

Because we used existing, available national-level data, our roadway design and land-use variables represent recent characteristics of the hot spot corridors. Therefore, there are likely to be some inconsistencies between our variable measurements and the true conditions present during the 2001-2008 period, in particular. This limitation is unlikely to impact our broad findings across the 60 corridors, but it prevented us from doing an in-depth comparison of how changes in specific corridor characteristics compared with changes in fatal crashes between the 2001-2008 and 2009-2016 time periods. Future research should gather more historic explanatory variable data to develop a more complete picture of how changes in hot spot characteristics relate to changes in fatal pedestrian crash outcomes over time.

Freeway crashes were prevalent in regions such as Dallas and Atlanta, but we did not include them in our hot spot analysis. People who get out of their cars on the freeway and then are hit are one particular type of fatality; people who walk/run across the freeway are another type of fatality (and more relevant to this type of analysis). To be conservative, we excluded all freeway crashes, as these two situations cannot readily be distinguished. However, future research should examine pedestrian fatalities while crossing at-grade freeways, such as the $900 \mathrm{~m}$ stretch between the St. Francis Avenue and S Buckner Boulevard overpasses along Interstate 30 in east Dallas, where 11 pedestrians were killed between 2009-2016.

Extensions of our work could test the sensitivity of the results to using different parameters for the hot spot analysis. For example:

- Use a different threshold of crashes to define a hot spot corridor. Setting the threshold below six fatal crashes would require additional resources.

- Consider different time periods. We chose eight years because it provided a larger number of crashes within specific corridors than three or five years. We also used this length because it was 
a simple way to split 16 years of available data. Several different hot spots would likely be identified if different eight-year periods were used (e.g., 2005-2012) or if a different number of years was used. However, it is likely that these corridors would generally have similar characteristics to the hot spots identified during 2001-2008 and 2009-2016.

- Test different corridor lengths (e.g., $500 \mathrm{~m}$ or $1500 \mathrm{~m}$ ). The hot spots identified using these distances could reveal additional insights about pedestrian fatality problems. For example, shorter lengths could identify problems associated with specific crossing locations while longer lengths could show corridors with consistent problems through multiple neighborhoods or communities.

Further research should also use available FARS data to examine characteristics of individual crashes within each corridor. For example, did certain corridors have higher proportions of fatal pedestrian crashes during darkness, at intersections, or involving turning vehicles? This information could be instructive for identifying specific countermeasures. Additionally, similar nationwide analyses should be conducted for fatal crashes involving automobile users, motorcyclists, and bicyclists to provide more complete information for vision zero efforts.

\section{Conclusion}

Pedestrian safety must be improved to create a future transportation system that produces zero deaths. Fatal pedestrian crashes are not random events. We found many similar characteristics among US fatal pedestrian crash hot spot corridors, including multilane roadways, high speed limits, high traffic volumes, adjacent commercial land uses, and nearby lower-income neighborhoods. Our results support a systemic approach to improve pedestrian safety: agencies should identify other roadway corridors with similar characteristics throughout the US and take actions to reduce the risk of future pedestrian fatalities. These actions include a combination of engineering, education, and automated enforcement treatments and strategies to provide safe, convenient pedestrian crossings and reduce vehicle speeds. Appropriate safety strategies will depend on specific roadway features and the surrounding land-use context; some corridors will require a long-term commitment to change development patterns. Ultimately, the engineering and planning professions must prioritize pedestrian safety over automobile mobility in order to save lives.

\section{Author contribution statement}

The authors confirm contribution to the paper as follows: study conception and design: Schneider, Sanders, Proulx; literature review: Schneider, Sanders; data collection: Schneider, Moayyed; analysis and interpretation of results: Schneider, Sanders, Proulx, Moayyed; draft manuscript preparation: Schneider, Sanders, Proulx, Moayyed. All authors reviewed the results and approved the final version of the manuscript.

\section{Appendices}

Appendices A and B can be found at https://www.jtlu.org/index.php/jtlu/article/view/1825. 


\section{References}

Ambros, J., Havránek, P., Valentová, V., Křivánková, Z., \& Striegler, R. (2016). Identification of hazardous locations in regional road network-Comparison of reactive and proactive approaches. Transportation Research Procedia, 14, 4209-4217.

American Association of State Highway Transportation Professionals (AASHTO). (2010). Highway safety manual (first ed.). Washington, DC: AASHTO

Blackburn, L., Zegeer, C., \& Brookshire, K. (2017). Guide for improving pedestrian safety at uncontrolled crossing locations (FHWA-SA-17-072). Washington, DC: Federal Highway Administration.

Boeing, G. (2017). OSMnx: New methods for acquiring, constructing, analyzing, and visualizing complex street networks. Computers, Environment and Urban Systems, 65, 126-139. https://doi. org/10.1016/j.compenvurbsys.2017.05.004

City of Oakland, California. (2017). 2017 City of Oakland pedestrian plan update. Retrieved from https://cao-94612.s3.amazonaws.com/documents/Ped-Plan-2017-rev-mar2018-edited-HIN.pdf

Clifton, K. J. \& Kreamer-Fults, K. (2007). An examination of the environmental attributes associated with pedestrian-vehicular crashes near public schools. Accident Analysis \& Prevention, 39(4), $708-715$.

Dai, D. (2012). Identifying clusters and risk factors of injuries in pedestrian-vehicle crashes in a GIS environment. Journal of Transport Geography, 24, 206-214.

DiMaggio, C., Mooney, S., Frangos, S., \& Wall, S. (2016). Spatial analysis of the association of alcohol outlets and alcohol-related pedestrian/cyclist Injuries in New York City. Injury Epidemiology, 3(1), 11.

Dumbaugh, E., \& Li, W. (2010). Designing for the safety of pedestrians, cyclists, and motorists in urban environments. Journal of the American Planning Association, 77(1), 69-88.

Ecola, L., Popper, S. W., Silberglitt, R., \& Fraade-Blanar, L. (2018). The road to zero: A vision for achieving zero roadway deaths by 2050, Rand Corporation, prepared for National Safety Council. Retrieved from https:/www.rand.org/content/dam/rand/pubs/research_reports/RR2300/RR2333/ RAND_RR2333.pdf

Elvik, R. (2008). A survey of operational definitions of hazardous road locations in some European countries. Accident Analysis \& Prevention, 40(6), 1830-1835.

Ewing, R., Schieber, R. A., \& Zegeer, C. V. (2003). Urban sprawl as a risk factor in motor vehicle occupant and pedestrian fatalities. American Journal of Public Health, 93(9), 1541-1545.

Ewing, R., \& Dumbaugh, E. (2009). The built environment and traffic safety: A review of empirical evidence. Journal of Planning Literature, 23(4), 347-367.

Geyer, J., Raford, N., Pham, T., \& Ragland, D. R. (2006). Safety in numbers: Data from

Oakland, California. Transportation Research Record: Journal of the Transportation Research Board, 1982, $150-154$.

Griswold, J., Fishbain, B., Washington, S., \& Ragland, D. R. (2011). Visual assessment of pedestrian crashes. Accident Analysis \& Prevention, 43(1), 301-306.

Governors Highway Safety Association (GHSA). (2019). Pedestrian fatalities by state: 2018 preliminary data, spotlight on highway safety. Retrieved from https://www.ghsa.org/resources/Pedestrians 19

IBM Corp. (2018). IBM SPSS statistics for Windows, Version 26.0. Armonk, NY: IBM Corp.

Jacobsen, P. L. (2003). Safety in numbers: More walkers and bicyclists, safer walking and bicycling. Injury Prevention, 9, 205-209.

Jang, K., Park, S. H., Kang, S., Song, K. H., Kang, S., \& Chung, S. (2013). Evaluation of pedestrian safety: Pedestrian crash hot spots and risk factors for injury severity. Transportation Research Record: 
Journal of the Transportation Research Board, 2393, 104-116.

Kim, K., \& Yamashita, E. Y. (2007). Using a K-means clustering algorithm to examine patterns of pedestrian involved crashes in Honolulu, Hawaii. Journal of Advanced Transportation, 41(1), 69-89.

Lee, C., \& Abdel-Aty, M. (2005). Comprehensive analysis of vehicle-pedestrian crashes at intersections in Florida. Accident Analysis \& Prevention, 37(4), 775-786.

Lefler, D. E., \& Gabler, H. C. (2003). The fatality and injury risk of light truck impacts with pedestrians in the United States. Accident Analysis and Prevention, 949, 1-10.

Loukaitou-Sideris, A., Liggett, R., \& Sung, H. (2007). Death on the crosswalk: A study of pedestrianautomobile collisions in Los Angeles. Journal of Planning Education and Research, 26, 338-351.

Mansfield, T., Peck, D., Morgan, D., McCann, B., \& Teicher, P. (2018). The effects of roadway and built environment characteristics on pedestrian fatality risk: A national assessment at the neighborhood scale. Accident Analysis and Prevention, 121, 166-176.

Manson, S., Schroeder, J., Van Riper, D., \& Ruggles, S. (2018). IPUMS national historical geographic information system: Version 13.0. Minneapolis: University of Minnesota. Retrieved from http:// https://doi.org/10.18128/D050.V13.0

Miranda-Moreno, L., Morency, P., \& El-Geneidy, A. (2011). The link between built environment, pedestrian activity and pedestrian-vehicle collision occurrence at signalized intersections. Accident Analysis \& Prevention, 43(5), 1624-1634.

Montella, A. (2010). A comparative analysis of hotspot identification methods. Accident Analysis \& Prevention, 42(2), 571-581.

Morency, P., \& Cloutier, M.-S. (2006). From targeted 'black spots' to area-wide pedestrian safety. Injury Prevention, 12, 360-364.

National Highway Traffic Safety Administration. (NHTSA). (1993). Traffic safety facts 1993: Pedestrians. Retrieved from https://crashstats.nhtsa.dot.gov/Api/Public/Publication/93F7

National Highway Traffic Safety Administration (NHTSA). (2007). Automated enforcement: A compendium of worldwide evaluations of results. Retrieved from http://www.nhtsa.gov/DOT/NHTSA/Traffic\%20Injury\%20Control/Articles/Associated\%20Files/HS810763.pdf

National Highway Traffic Safety Administration (NHTSA). (2018). Fatality analysis reporting system (FARS) analytical user's manual, 1975-2017 (DOT HS 812 602). Washington, DC: NHTSA.

National Highway Traffic Safety Administration (NHTSA). (2019a). Fatality analysis reporting system (FARS) encyclopedia, FARS data tables. Retrieved from https:/www-fars.nhtsa.dot.gov/Main/index.aspx.

National Highway Traffic Safety Administration (NHTSA). (2019b). Traffic safety facts, 2017 data: Pedestrians (DOT HS 812 681). Washington, DC: NHTSA.

National Transportation Safety Board (NTSB). (2019). Accidents involving passenger fatalities: US airlines (Part 121) 1982 - present. Retrieved from https:/www.ntsb.gov/investigations/data/Pages/ paxfatal.aspx.

Owens, D., \& Sivak, M. (1993). The role of reduced visibility in nighttime road fatalities, University of Michigan Transportation Research Institute (UMTRI-93-33). Retrieved from https://deepblue.lib. umich.edu/bitstream/handle/2027.42/49541/UMTRI-93-33.pdf?sequence=1\&isAllowed=y

Owens, J. M., Dingus, T. A., Guo, F., Fang, Y., Perez, M., McClafferty, J., \& Tefft, B. (2018). Prevalence of drowsy driving crashes: Estimates from a large-scale naturalistic driving study (research brief). Washington, DC: AAA Foundation for Traffic Safety.

Rankavat, S., \& Tiwari, G. (2013). Pedestrian accident analysis in Delhi using GIS. Journal of the Eastern Asia Society for Transportation Studies, 10, 1446-1457.

Schneider, R. J., Khattak, A. J. \& Zegeer, C. V. (2002). A proactive method of improving pedestrian 
safety using GIS: Example from a college campus. Transportation Research Record: Journal of the Transportation Research Board, 1773, 97-107.

Schneider, R. J., Ryznar, R. M., \& Khattak, A. J. (2004). An accident waiting to happen: A spatial approach to proactive pedestrian planning. Accident Analysis and Prevention, 36, 193-211.

Schneider, R. J., Vargo, J., \& Sanatizadeh, A. (2017). Comparison of US metropolitan region pedestrian and bicyclist fatality rates. Accident Analysis and Prevention, 106, 82-98.

Schuurman, N., Cinnamon, J., Crooks, V. A., \& Hameed, S. M. (2009). Pedestrian injury and the built environment: An environmental scan of hotspots. BMC Public Health, 9. https://doi. org/10.1186/1471-2458-9-233

Siddiqui, N. A., Chu, X., \& Guttenplan, M. (2006). Crossing locations, light conditions, and pedestrian injury severity. Transportation Research Record: Journal of the Transportation Research Board, 1982, 141-149.

Smart Growth America. (2014). Measuring sprawl 2014. Retrieved from https://www.smartgrowthamerica.org/app/legacy/documents/measuring-sprawl-2014.pdf

Smart Growth America and National Complete Streets Coalition. (2017). Dangerous by design 2016. Retrieved from https://smartgrowthamerica.org/resources/dangerous-by-design-2016/

Solano County, California. (2018). 2018 Solano County travel safety plan. Retrieved from https://sta. ca.gov/wp-content/uploads/2019/01/Solano-Travel-Safety-Plan-2018.pdf

Stimpson, J. P., Wilson, F. A., \&. Muelleman, R. L. (2013). Fatalities of pedestrians, bicycle riders, and motorists due to distracted driving motor vehicle crashes in the US, 2005-2010. Public Health Reports, 128(6), 436-442.

Stoker, P., Garfinkel-Castro, A., Khayesi, M., Odero, W., Mwangi, M. N., Peden, M., \& Ewing, R. (2015). Pedestrian safety and the built environment: A review of the risk factors. Journal of Planning Literature, 30(4), 377-392.

Sullivan, J. M., \& Flannagan, M. J. (2001). The role of ambient light level in fatal crashes: Inferences from daylight saving time transitions. Accident Analysis \& Prevention, 34(4), 487-498.

Tang, X., Eftelioglu, E., Oliver, D., \& Shekhar, S. (2017). Significant linear hotspot discovery. IEEE Transactions on Big Data, 3(2).

Tefft, B. (2013). Impact speed and a pedestrian's risk of severe injury or death. Accident Analysis and Prevention, 50, 871-878.

Thomas, L., Lan, B., Sanders, R. L., Frackelton, A., Gardner, S., \& Hintze, M. (2017). Changing the future? Development and application of pedestrian safety performance functions to prioritize locations in Seattle, WA. Transportation Research Record: Journal of the Transportation Research Board, 2659, 212-223.

Thomas, L., Sandt, L., Zegeer, C., Kumfer, W., Lang, K., Lan, B., ... \& Schneider, R. J. (2018). Systemic pedestrian safety analysis (National Cooperative Highway Research Program, report 893). Washington, DC: National Cooperative Highway Research Program.

Toward Zero Deaths, National Strategy on Highway Safety. (2011). American Association of State Highway and Transportation Officials toward zero deaths resolution. Retrieved from http://www. towardzerodeaths.org/resource/aashto-toward-zero-deaths-resolution/

Van Houten, R., Malenfant, L., Blomberg, R. D., \& Huitema, B. E. (2017). The effect of high-visibility enforcement on driver compliance with pedestrian right-of-way laws: 4-year follow-up. Washington, DC: National Highway Traffic Safety Administration.

Vision Zero Network. (2019). Vision zero cities. Retrieved from https://visionzeronetwork.org/about/ vision-zero-network/elevating-efforts-in-vision-zero-cities-across-the-u-s/

Wedagama, D. P., Bird, R. N., \& Metcalfe, A. V. (2006). The influence of urban land-use on non- 
motorized transport casualties. Accident Analysis \& Prevention, 38(6), 1049-1057.

Wier, M., Weintraub, J., Humphreys, E. H., Seto, E., \& Bhatia, R. (2009). An area-level model of vehicle-pedestrian injury collisions with implications for land use and transportation planning. $A c-$ cident Analysis \& Prevention, 41(1), 137-145.

US Census Bureau. (2019). 2018 TIGER/line shapefiles. Retrieved from https://www2.census.gov/geo/ tiger/TIGER2018/ROADS/

Zegeer, C. V., Nabors, D., Lagerwey, P., Sundstrom, C., Lovas, D., Huber, T., ... \& Bushell, M. (2013). PEDSAFE: Pedestrian safety guide and countermeasure selection system. Washington, DC: Federal Highway Administration.

Zegeer, C., Srinivasan, R., Lan, B., Carter, D. Smith, S., Sundstrom, C., ... \& Van Houten, R. (2016). Development of crash modification factors for uncontrolled pedestrian crossing treatments (National Cooperative Research Program, report 841). Washington, DC: NCRP. 